\title{
Thermal Response Behavior of a Photo-Crosslinked Liquid Crystalline Polymer and a Side Chain Liquid Crystalline Polymer
}

\author{
Ryoma Amanuma*, Ayumi Kobayashi, Kohei Iritani, and Takashi Yamashita ${ }^{* *}$ \\ Department of Applied Chemistry, School of Engineering, \\ Tokyo University of Technology, 1404-1 Katakura, Hachioji, Tokyo 192-0982, Japan \\ *g511590020d@edu.teu.ac.jp \\ **yamashitatks@stf.teu.ac.jp
}

\begin{abstract}
Liquid crystalline polymers (LCPs) have attracted much attention because of their macroscopic deformation to external stimuli such as heat or light-irradiation. Among them, thermal responsible polymers, in which their macroscopic structures are controlled by heat treatment, are one of the most attractive materials. Although the thermal responsive materials by thermal phase transition were developed in wide for the application to the electronic devices and sensors, few examples have been applied to practical uses because of their high transition temperatures. In this paper, the development of LCPs with low deformation temperatures was investigated aiming for the application to functional fibers responding to body temperature. The phase transition temperature was found to depend on the content of crosslinking unit, and the film deformation was controlled by the one-side photo-irradiation to change the degree of crosslinking between two sides.
\end{abstract}

Keywords: Actuator, Liquid crystalline polymer, Phase transition, Photo-crosslinking, Thermal responsibility

\section{Introduction}

Recently, stimuli-responsive materials in which reversible expansion and contraction or bending and tightening induced by external stimuli, such as heat, light, electricity, and magnetism have been intense interest because of their potential for their uses in artificial muscles, actuators, and chemical sensors [1-4]. In 1997, P. G. de Gennes et al. demonstrated the flexible molding of liquid crystalline elastomers (LCEs) by temperature controlling, lightirradiation, and applying electricity and magnetism for application to artificial muscles [5]. In addition, we previously reported that the photo-induced volume control using polymers including an azobenzene unit, in which phase transition between nematic and isotropic phases induced by UVirradiation, was achieved [6].

Regarding to the molecular design for the fabrication of stimuli-responsive materials, there are two key factors. One is the cross-link formation between polymer chains. It is expected that the cross-linking realizes the preparation of monodomain alignment, which achieves a freestanding actuator. In the past, based on the photoinduced cross-linking, reversible deformation of complex 3D structures of materials was performed $[7,8]$. The other is the adjustment of the length of side chains of polymers, which affects the phase transition temperature [9]. For example, it was revealed that relatively low phase transition temperature at less than $40{ }^{\circ} \mathrm{C}$ was displayed using cyanobiphenyl units having short alkyl chains such as $\mathrm{C}_{6}$ and $\mathrm{C}_{8}$ chains [10].

Although the application of the thermal or light responsive materials for fibers and films aiming to electronic devices and sensors was reported, to the best of our knowledge, there is no example of the fabrication of daily necessities such as clothes. In this report, we purposed the development of functional fibers using LCEs, which display thermal responsiveness at around human body temperature. We expected that the air permeability of the clothes formed by such fibers would be controlled by the deformation of the thermal responsible LCEs. That 
is, when our body temperatures increase because of exercises or sickness, clothes are ventilated due to the contraction of LCEs in the fibers. To this end, we designed liquid crystal polymers (LCPs), CBPA and CAPE. Figures $1 \mathrm{a}$ and $1 \mathrm{~b}$ show chemical reactions in the syntheses of the polymers. The former is prepared by copolymerization of methacrylic acid alkoxybiphenyl (C3M-CB and C6M-CB), bis(methacylic acid hexyloxy)biphenyl (BMHBP), and butyl methacrylate (BM), and the latter is copolymerized by bis(hydroxylhexyloxy)biphenyl (BHHBP), phenylsuccinic acid (PSA), and hydroxyhexyloxy coumaric acid (6HCA). For CBPA, BMHBP is included as a cross-linking unit. In addition, $\mathrm{C}_{3}$ and $\mathrm{C}_{6}$ alkyl chains in $\mathbf{C 3 M - C B}$ and

(a)

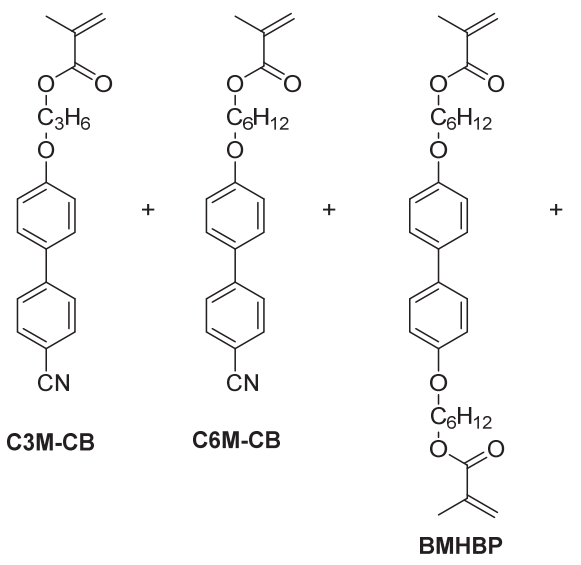

C6M-CB are adopted as side chains for the lowering the phase transition temperature. Moreover, MB is introduced because of the possibility for improvement of flexibility and lowering phase transition temperature of polymers. In the case of CAPE, PSA is introduced for the construction of a helical structure in the polymer chains. Furthermore, 6HCA having a coumarate unit is adopted as a reactive site by photo-irradiation after the molding because it would bind to each other in head-to-head and head-to-tail manners to form a cyclobutane ring [11] as shown in Fig. 1c. Herein, we evaluated the thermophysical properties with different molar ration of monomers.

(b)
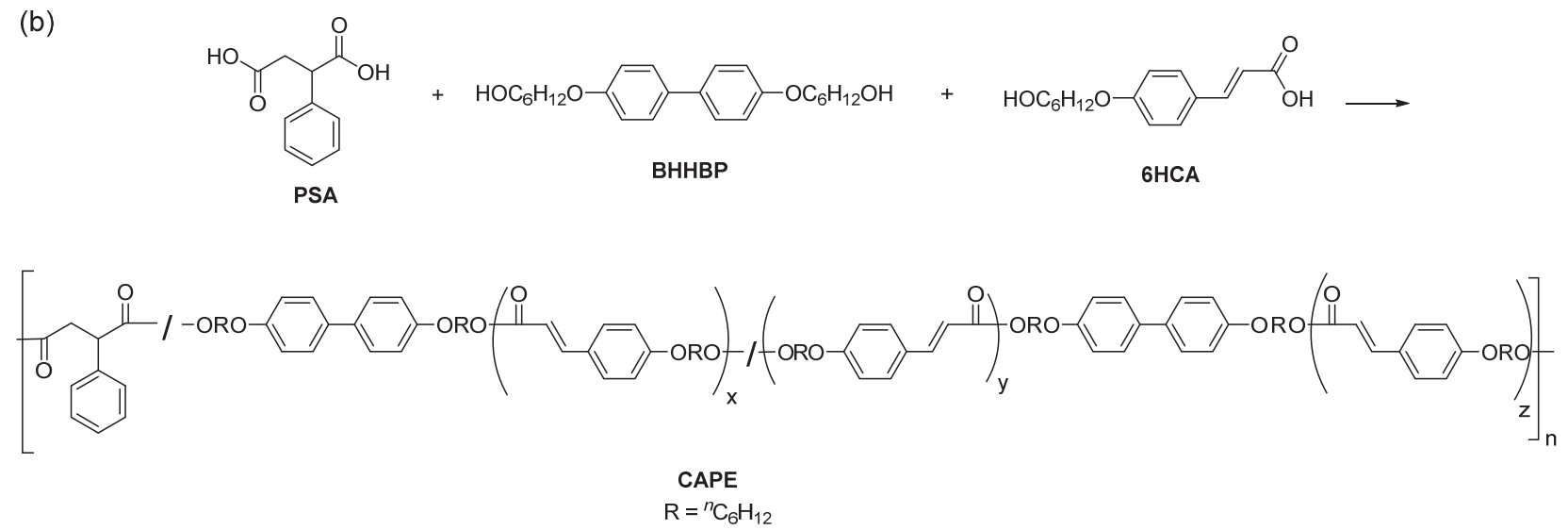

(c)
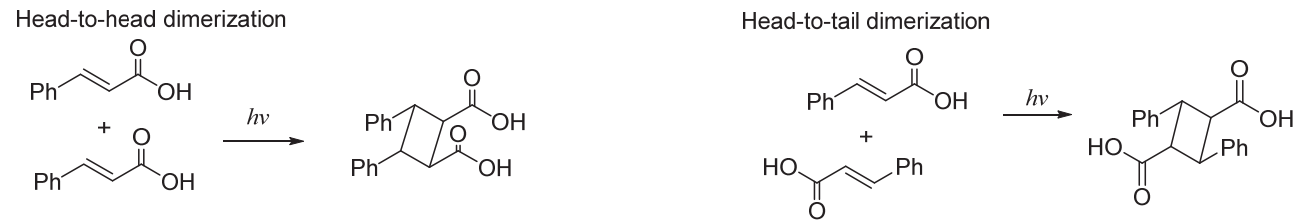

Fig. 1. Chemical reactions in syntheses of CBPA (a) and CAPE (b), and photocycloaddition reaction of coumaric acid in head-to-head and head-to-tail manners (c). 


\section{Experimental}

\subsection{Apparatus}

For differential thermal analysis (DTA), a thermogravimetry/differential thermal analyzer (DTA-60, Shimadzu Corporation) was used at heating rate of $1{ }^{\circ} \mathrm{C} / \mathrm{min}$. For differential scanning calorimetry (DSC), a differential scanning calorimeter (Thermo plus EVO2, Rigaku Corporation) was used at a heating rate of $5{ }^{\circ} \mathrm{C} / \mathrm{min}$ using $5 \mathrm{mg}$ of samples. Polarizing microscopy (PO) observations were performed by using a microscope (CX31PF, OLYMPUS). UV-vis absorption spectra were recorded on a spectrometer (V-550, JASCO) in the rage of wavelength at $200-800 \mathrm{~nm} .{ }^{1} \mathrm{H}-\mathrm{NMR}$ $(400 \mathrm{MHz})$ spectra were mesured on a Buruker UlreaShield Plus 400 spectrometer. The spectra were referenced to a proton signal of tetramethylsilane $(0 \mathrm{ppm})$ in DMSO- $d_{6}$ and $\mathrm{CDCl}_{3}$. The heating of LCPs was performed by a hot stage (FP82HT, METTLER) with a thermos system controller (FP90, METTLER) to set temperatures above $5{ }^{\circ} \mathrm{C}$ from the phase transition temperature in each sample.

\subsection{Reagents}

All commercially available reagents were used as received from TCI or Wako Pure Chemical Industries, Ltd. N,N-Dimethylformsmide (DMF) and tetrahydrofuran (THF) were dried over molecular sieves $4 \mathrm{~A}$ before use. Other solvents were used without purifications.

\subsection{Preparation of films}

All sample films were prepared by compression after samples were sandwiched between polypropylene films with heating at $100{ }^{\circ} \mathrm{C}$ for CBPA and $70^{\circ} \mathrm{C}$ for CAPE.

\subsection{Photo-irradiation to films}

To obtain cross-linked materials, films were irradiated by UV light using a Xenon lamp with a filter cutting off wavelength less than $320 \mathrm{~nm}$. The one side of films were exposed to the UV light.

\subsection{Syntheses of polymers}

\subsubsection{General procedure of synthesis of CBPA}

C6M-CB (668 mg, $2.84 \mathrm{mmol}$ ), C3M-CB (590 $\mathrm{mg}, 2.84 \mathrm{mmol})$, and AIBN (14.7 mg, $89.0 \mathrm{mmol})$ were dissolved in THF $(5.0 \mathrm{~mL})$. After stirring at $50{ }^{\circ} \mathrm{C}$ for $1.5 \mathrm{~h}, 55^{\circ} \mathrm{C}$ for $0.5 \mathrm{~h}, 60^{\circ} \mathrm{C}$ for $0.5 \mathrm{~h}$, $70{ }^{\circ} \mathrm{C}$ for $3 \mathrm{~h}, 75^{\circ} \mathrm{C}$ for $1 \mathrm{~h}, 90^{\circ} \mathrm{C}$ for $0.5 \mathrm{~h}$, the solution was added to methanol dropwise for reprecipitation. After the filtration, product was dried under vacuum for overnight. CBPA-2 (1.09 g) was obtained as a white solid.

\subsubsection{General procedure of synthesis of CAPE}

BHHBP (4.51 g, $11.7 \mathrm{mmol})$, 6HCA $(1.29 \mathrm{~g}$, $3.89 \mathrm{mmol})$, PSA (2.27 g, $11.7 \mathrm{mmol}), \mathrm{Zn}(\mathrm{AcO})_{2}$ (23.4 mg, $128 \mu \mathrm{mol})$, and $\mathrm{Sb}_{2} \mathrm{O}_{3}(23.4 \mathrm{mg}, 80.3$ $\mu \mathrm{mol})$ were placed to a $100 \mathrm{~mL}$ round-flask. After the mixture was stirred and heated at $180{ }^{\circ} \mathrm{C}$ for $1 \mathrm{~h}$ under vacuum, reaction temperature increased to $200{ }^{\circ} \mathrm{C}$ at the ratio of $20{ }^{\circ} \mathrm{C} / \mathrm{h}$. After stirring at $200{ }^{\circ} \mathrm{C}$ for $4.5 \mathrm{~h}$, the mixture was cooled to room temperature under vacuum. The crude product was dissolved in THF and the solution was added to water dropwise for reprecipitation. After the filtration, CAPE-3 (7.33 g) was gave as a white solid.

\section{Results and discussion}

\subsection{Syntheses of polymers}

CBPA [12] and CAPE [13,14] were synthesized by radical polymerization using AIBN as a radical initiator and by polycondensation under solventfree condition, respectively, through modification of the previously reported methods. To investigate the effect of molar ratio of monomers for the thermal responsibility of polymers, we synthesized series of CBPA and CAPE with different monomer

Table 1. Molar ratio of monomers for series of CBPA.

\begin{tabular}{lcccc}
\hline Polymer C3M-CB C6M-CB BMHBP & BM \\
\hline CBPA-1 & - & 1 & - & - \\
CBPA-2 & 1 & 1 & - & - \\
CBPA-3 & 1 & 1 & 2 & - \\
CBPA-4 & 5 & 5 & 1 & - \\
CBPA-5 & 25 & 25 & 1 & - \\
CBPA-6 & 100 & 100 & 1 & - \\
CBPA-7 & 150 & 150 & 1 & - \\
CBPA-8 & 25 & 25 & 1 & 25 \\
\hline
\end{tabular}

Table 2. Molar ratio of monomers for series of CAPE.

\begin{tabular}{cccc}
\hline Polymer & BHHBP PSA & 6HCA \\
\hline CAPE-1 & 1 & 1 & 0.091 \\
CAPE-2 & 1 & 1 & 0.11 \\
CAPE-3 & 1 & 1 & 0.33 \\
CAPE-4 & 1 & 1 & 0.2 \\
CAPE-5 & 1 & 1 & 1 \\
CAPE-6 & 1 & 1 & 2 \\
\hline
\end{tabular}


composition ratios. The molar ratio of the monomer component of CBPA and CAPE are summarized in Tables 1 and 2, respectively. Note that, for CBPA-3 and CBPA-8, gel-like products were obtained after the polymerization.

\subsection{Thermal analyses of series of CBPA}

Thermal analyses of the series of CBPA were performed by DSC measurements. Figures 2 and 3 show DSC curves of each sample and typical polarizing microscopy (PO) images of samples observed on the hot stage at temperatures inserted in the images. For CBPA-1 and CBPA-2, which are a homopolymer of C6M-CB and a copolymer including C3M-CB and C6M-CB, a peak was observed at ca. $90{ }^{\circ} \mathrm{C}$ during both of raising and lowering temperature processes. Based on $\mathrm{PO}$ images obtained at 101 and $102{ }^{\circ} \mathrm{C}$ (Fig. 3a), it was found that the peak in DSC curve was corresponded to the liquid crystal phase transition. In the cases of CBPA-4-7, several peaks were observed in the scanning range. On the basis of $\mathrm{PO}$ observations, we considered that peaks detected at ca. $100{ }^{\circ} \mathrm{C}$ were assigned to the liquid crystal phase transition, which are slightly higher compared to those of CBPA-1 and CBPA-2. Figure $3 \mathrm{~b}$ shows PO images of CBPA-4 as an example. This result suggests that
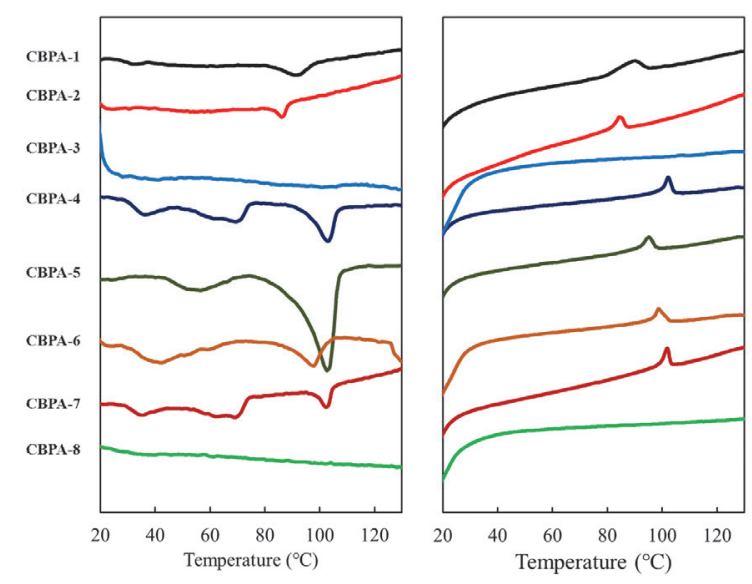

Fig. 2. DSC curves during the process of raising (left) and lowering (right) for the series of CBPA.

(a)

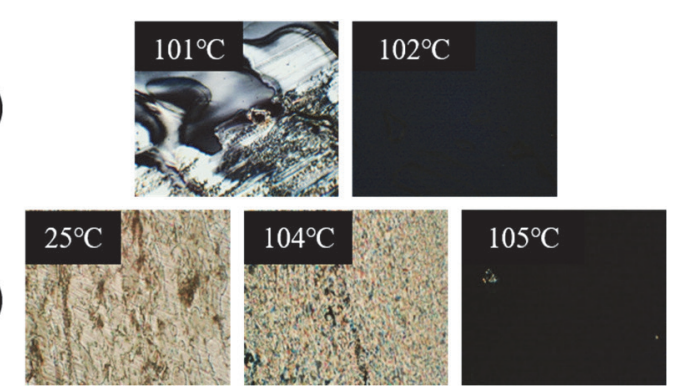

Fig. 3. PO images of films formed by CBPA-1 (a) and CBPA-4 (b). addition of a small amout of BMHBP in polymer raise the liquid crystal phase transition temperature probably because of increasing molar wight of polymers due to the cross-linking. Other endothermic peaks indicate the presence of several thermal stable phases within the liquid crystal phase as shown in Fig. $3 \mathrm{~b}\left(25\right.$ and $\left.104^{\circ} \mathrm{C}\right)$. On the other hand, in the cases of CBPA-3 and CBPA-8, no peak was observed during temperature scanning. These are probably due to, for the former, a large amount of the cross-linking unit, and the relatively higher flexibility induced by the BM unit for the latter. Although it was found that LCPs including the cross-linking unit exhibits the liquid crystal phase transition, their temperatures are considerably higher than human body temperature.

\subsection{Macroscale thermal responses of series of CBPA.}

We investigated the thermal responsibility of the series of CBPA in macroscale. After the preparation of films using LCPs by heating compressionmolding, we performed heating experiment of the films on the hot stage in which temperature was set to above $5{ }^{\circ} \mathrm{C}$ from each phase transition temperature detected by DSC. Films obtained from CBPA-1 and CBPA-2 were fragile and melted during heating, and the gelled CBPA-3 was too hard to form a film. For CBPA-4-7, off-white flexible films were formed. Although the flexibility of the film was improved by the addition of the crosslinking unit, these films melted with heating. As an example, photo images of the film formed by CBPA-5 without and with heating at $108{ }^{\circ} \mathrm{C}$ were shown in Fig. 4a. In the case of a film of CBPA-8, although gel-like product was obtained by polymerization, the flexible film was formed and it

(a)
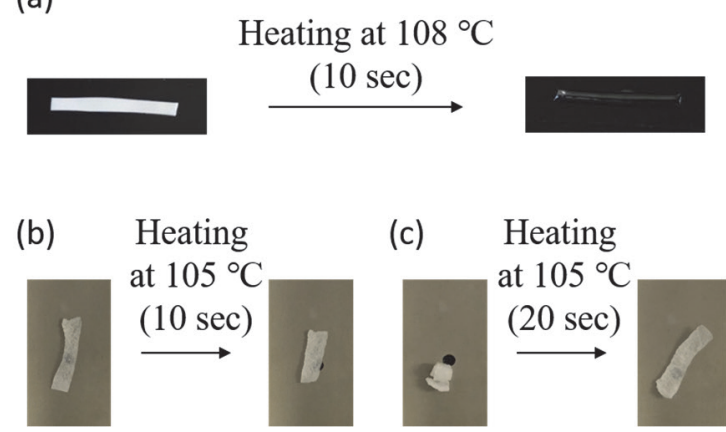

Fig. 4. (a) Photo images of the film formed by CBPA5 before (left) and after (right) heating at $108{ }^{\circ} \mathrm{C}$ for 10 sec. (b, c) Photo images of the film formed by CBPA-8 without and with heat at $105^{\circ} \mathrm{C}$ for $10 \mathrm{sec}$ (b), and that after stretching without and with heat at $105^{\circ} \mathrm{C}$ for $20 \mathrm{sec}(\mathrm{c})$. 
exhibited heat resistance at least $105^{\circ} \mathrm{C}$ (Fig. 4b). After cooling of the film to room temperature, it was artificially stretched to form a curled film. As shown in Figure $4 \mathrm{c}$, the shape of the film was changed to that before stretching, which is indicated that the film exhibited shape memory character with heating by including the $\mathbf{B M}$ unit in the polymer.

\subsection{Thermal properties of CAPE}

Next, we performed thermal analyses using the series of CAPE in which the mixing molar ratios of 6HCA was set to be from $50 \%$ to $9 \%$. In Fig. 5, DTA curves of the samples are shown. In the cooling process of the samples of CAPE-1-4, two peaks at around $50{ }^{\circ} \mathrm{C}$ and $60{ }^{\circ} \mathrm{C}$ were observed. As an example, Fig. 6a shows PO images of CAPE-3 obtained on the hot stage set at 30,52 , and $58^{\circ} \mathrm{C}$. From the images, it was revealed that the peak at higher temperature is due to the liquid crystalline phase transition. With increasing the molar ratio of 6HCA, the liquid crystal phase transition temperature was shifted to lower. Unfortunately, we did not understand completely on the phase transition at lower temperature because PO images

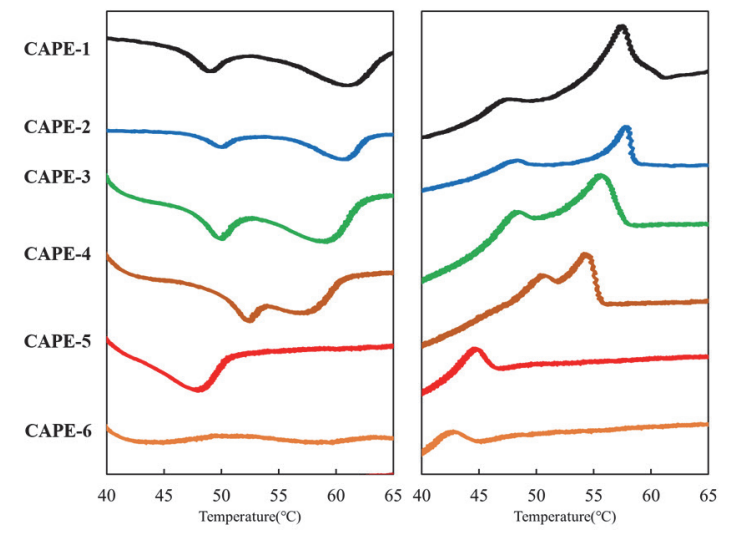

Fig. 5. DTA curves during the process of raising (left) and lowering (right) for the series of CAPE.

(a)

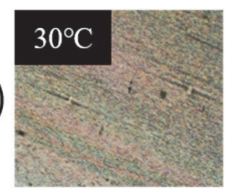

(b)

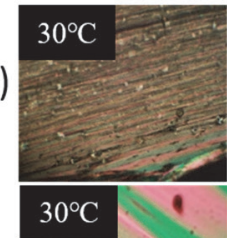

(c)
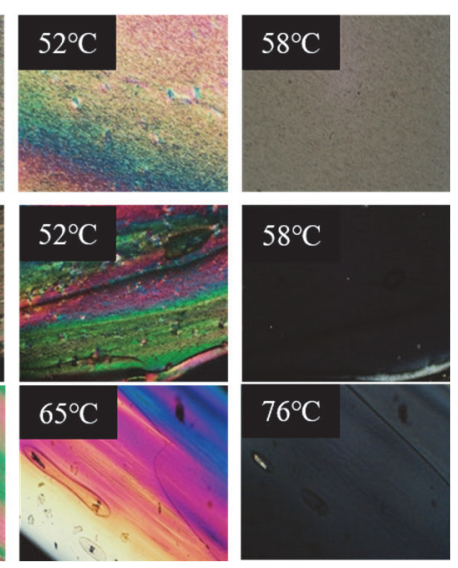

Fig. 6. PO images of films by CAPE-3 (a), CAPE-5 (b), and CAPE-6 (c). and motional chanses of films in macroscale were not observed. In DTA curves of CBPE-5, only one peak at ca. $48{ }^{\circ} \mathrm{C}$ was detected. This is due to the liquid crystal phase transition as shown in Figure $6 \mathrm{~b}$. For CAPE-6, quite weak peaks at. $43{ }^{\circ} \mathrm{C}$ and $60^{\circ} \mathrm{C}$ were observed. The latter is corresponded to the liquid crystalline phase transition (Fig. 6c). From these experiments, it was found that the presence of the 6HCA unit affected to lowering the temperature of liquid crystal phase transition although, unfortunately, the temperatures are higher by ca. $20^{\circ} \mathrm{C}$ in comparison with human body temperature.

\subsection{Cross-link formation of series of CAPE by UV- irradiation}

Next, we performed the cross-link formation of the series of CAPE by UV-irradiation using their thin films. Before UV-irradiation, the films were stretched in one direction to set to be ca. $50 \mu \mathrm{m}$ thickness in order to form the film with uniaxially oriented polymer chains. These films were demonstrated UV-irradiation with light at $320 \mathrm{~nm}$. Monitoring the cross-link formation was performed by measurements of absorption of the films. As an example, Fig. 7 shows UV spectra of the film formed by CAPE-3 before and after UV-irradiation for up to $180 \mathrm{~min}$. Before the UV-irradiation, the absorption at around $320 \mathrm{~nm}$, which is due to $\pi-\pi^{*}$ transition of the courmate unit, was observed. The absorbance decreased with UV-irradiation, and after the irradiation for $180 \mathrm{~min}$, it disappeared. This indicates the formation of a cycloubutane ring by the connection of the coumrate unit, in which the expected chemical reactions were shown in Fig. 1c although it was too difficult to refer to reaction types,

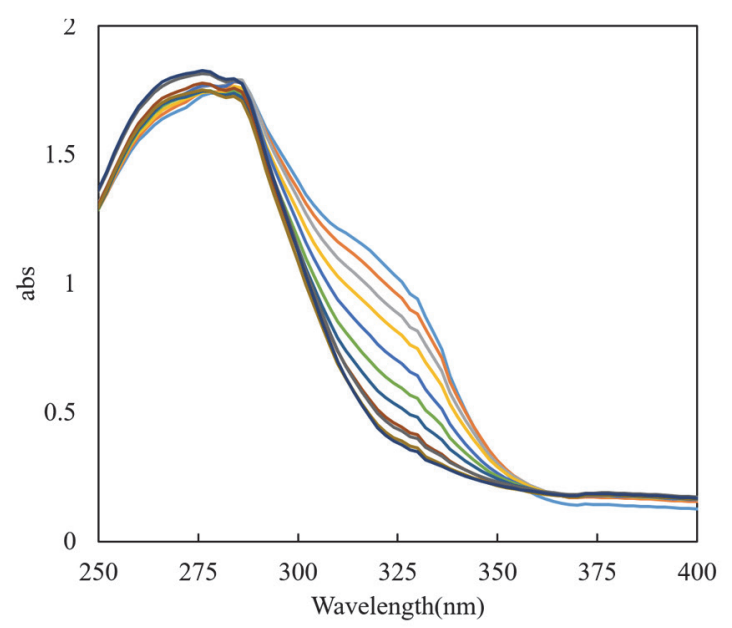

Fig. 7. UV spectra of the film formed by CPAE-3 before and after UV-irradiation at $320 \mathrm{~nm}$ for up to $180 \mathrm{~min}$. 
that is, head-to-head and head-to-tail manners. As a result, we concluded that crosslinked materials were obtained by the UV-irradiation.

3.6. Macroscale observations of thermal response of the film before and after cross-linking

We performed heating experiments of the film before and after the cross-linking. The left image of Fig. 8a shows a photo image of the non-crosslinked film of CAPE-3 fixed on the hot stage by a tape. We stretched the film to one direction before the crosslinking as shown in the middle of Fig. 8a. After heating at $65^{\circ} \mathrm{C}$ for $45 \mathrm{sec}$, the stretched film was shrunk in the vertical direction, and its shape changed to that before stretching (the right image of Fig. 8a), which is indicated that shape memory characteristic was exhibited. On the other hand, Fig. $8 \mathrm{~b}$ exhibits the photo image of the film, which was performed the UV-irradiation to one side of the film with the light at more than $320 \mathrm{~nm}$ for $90 \mathrm{~min}$, before and after heating at $65^{\circ} \mathrm{C}$ for $90 \mathrm{sec}$ on the hot stage. Surprisingly, the film showed a curled structure, in which the UV-irradiated side was bent inward with heating. These results suggested that cross-linked polymers shrank more efficiency compared to that without the cross-linking by heating treatment compared to that without the cross-linking because of effective entropy elasticity.

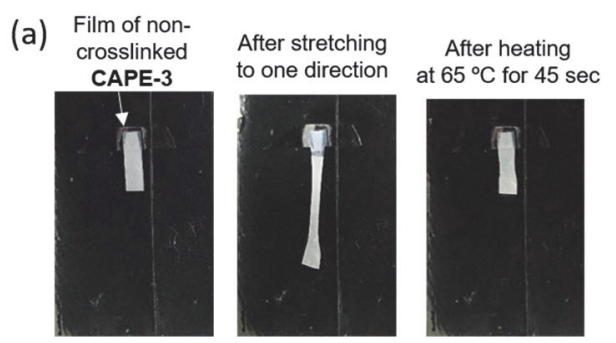

(b)

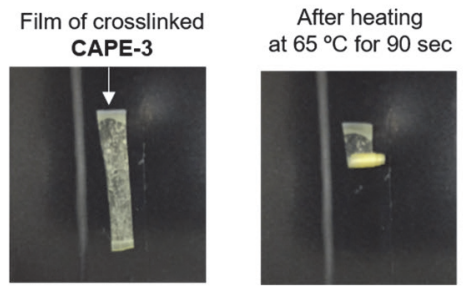

Fig. 8. Photo images of the films formed by CAPE3 before (a) and after (b) the cross-linking without and with heat at $65^{\circ} \mathrm{C}$ for the time inserted.

\section{Conclusion}

In order to decrease the phase transitional temperature to human body temperature, two types of liquid crystalline polymers, CBPA and CAPE, including cross-linking units and short alkyl chains with different molar ratios of monomers were synthesized. In the cases of CBPA having a small molar ratio of BMHBP, which is a cross-linking unit, it was found that, based on thermal analyses and PO observations, the phase transitional temperature was detected at more than $100{ }^{\circ} \mathrm{C}$, which is significantly higher than the temperature of human body. In addition, CBPA having a MB unit exhibited a shape memory characteristic. For series of CAPE, the phase transition was observed at lower compared to that of CBPA due to crosslinking using a 6HCA unit. In addition, it revealed that a film formed by CAPE, in which UV light irradiation was performed to one side, was curled because of the differentiation of the contraction of polymers before and after the cross-linking. Although it is necessary to design the polymer structure more precisely to achieve the phase transition at human body temperature, we found characteristic structural changes of LCPs in macroscale.

\section{References}

1. T. Ikeda, J. Mamiya, and Y. L. Yu, Angew. Chem. Int. Ed., 46 (2007) 506.

2. C. Ohm, M. Brehmer, and R. Zentel, Adv. Mater., 22 (2010) 3366.

3. X. Pang, J. Lv, C. Zhu, L. Qin, and Y. Yu, $A d v$. Mater, 31 (2019) 1904224.

4. F. Ge and Y. Zhao, Adv. Funct. Mater, 30 (2020) 1901890.

5. P. G. de Gennes, M. Hebert, and R. Kant, Phys. Macromol. Symp., 113 (1997) 39.

6. K. Okano, M. Shinohara, and T. Yamashita, Chem. Eur. J., 15 (2009) 3657.

7. J. I. Mamiya, A. Yoshitake, M. Kono, Y. Yu, and T. Ikea, J. Master. Chem., 18 (2008) 63.

8. Z. Cheng, S. Ma, Y. Zhang, S. Huang, and H. Yu, Macromolecules, 50 (2017) 8317.

9. N. Lebovka, V. Melnyk, Y. Mamunya, G. Klishevich, A. Goncharuk, and N. Pivovarava, Phys. E, 52 (2013) 65.

10. K. M. Lee and C. D. Han, Macromolecules, 36 (2003) 8796.

11. R. D. Hartley, W. H. Morrison III, F. Balza, and G. H. Neil Towers, Phytochemistry, 29 (1990) 3699.

12. Y. Zhan, G. Du, S. Yang, C. Xu, M. Lu, Z. Liu, and J. Y. Lee, ACS Appl. Mater. Interfaces, 7 (2015) 23.

13. R. Yang and Y. Zhao, Angew. Chem. Int. Ed., 56 (2017) 45.

14. H. Li, R. Yang, Y. Peng, H. Cao, and J. Li, J. Polym. Sci. B. Polym. Phys., 57 (2019) 14. 